VOLUME 23 (2018) 1-24

DOI: $10.24330 /$ ieja.373631

\title{
ALGEBRAS RELATED TO A CLASS OF 3-BLOCKS OF DEFECT 2
}

\author{
Burkhard Külshammer, Pierre Landrock and Stephanie Reinhardt \\ Received: 15 March 2016; Revised: 17 June 2017; Accepted: 11 September 2017 \\ Communicated by A. Çiğdem Özcan
}

\begin{abstract}
Motivated by a problem concerning the structure of certain 3blocks of defect 2 in finite groups we investigate a class of local algebras of dimension 9 over a field of characteristic 3. In particular, we compute the complexity of the unique simple module for any such algebra.
\end{abstract}

Mathematics Subject Classification (2010): 20C05, 20C20, 16L60

Keywords: Block, defect, symmetric algebra, complexity

\section{Introduction}

In this note we will consider certain 3-blocks with an elementary abelian defect group $D$ of order 9 . Such blocks were first studied systematically by Kiyota [4]. He identified 11 different types of these blocks, depending on the structure of the relevant inertial quotient, its action on $D$ and a certain 2-cocycle. For some cases he determined the numbers of irreducible ordinary and Brauer characters in these blocks; for other cases these are unknown even today.

Here we will be concerned with one of these 11 types. In this case the block $B$ is nonnilpotent, its inertial quotient is a Klein four group, and it contains a unique irreducible Brauer character and precisely 6 irreducible ordinary characters. In the following, we consider $B$ as an algebra over an algebraically closed field $k$ of characteristic 3 . Then the basic algebra $A$ of $B$ is a symmetric local $k$-algebra of dimension 9 which has a center of dimension 6 . Moreover, $A$ and $B$ are, of course, Morita equivalent. The isomorphism type of $A$, and thus the Morita equivalence class of $B$, was determined by Kessar in [3].

It is the purpose of this paper to revisit some of her arguments and to provide variations and simplifications of her methods. Some of these simplifications come from additional properties of the blocks in question which were not used in [3]. We hope that our variations may prove to be useful in more general situations. Some of our methods are inspired by [11], the master's thesis of the third author written under the guidance of the first author. 
We also point out a little mistake in Proposition 5.5 of [3]. However, Kessar assures us that this mistake does not influence the correctness of the main result in [3]. The starting point of Kessar was as follows. Suppose that $G$ is a finite group such that the group algebra $k G$ has a block $B$ with elementary abelian defect group $D$ of order 9. Let $b$ be the Brauer correspondent of $B$ in $N_{G}(D)$, again a block with defect group $D$. Using a result of the first author [7], she shows that the basic algebra $C$ of $b$ has generators $x, y$ and defining relations $x^{3}=y^{3}=x y+y x=0$ (cf. p. 491 of [3]). Her goal is then to show that $A$ and $C$ are isomorphic. This then ensures that $B$ and $b$ are Morita equivalent.

A result by Puig and Usami [10] shows that $B$ and $b$ are perfectly isometric. This implies that $A, B, b$ and $C$ have isomorphic centers; in particular, we have $Z(A) \cong Z(C)$. In order to show that $A \cong C$ we are going to use the following fact: $a^{3} \in K(A)=[A, A]$, for $a \in J(A)$. This holds because the defect group $D$ of $B$ has exponent 3, as will be explained in more detail in Section 2 below. This property was not used in [3]; it simplifies some of the proofs and some of the defining relations of our algebras.

We also use the fact that finitely generated modules over group algebras have a finite complexity (cf. [1]). As the referees of an earlier version of this paper pointed out, this is the first instance where this method is used to show that certain algebras cannot be blocks of finite groups. We hope that it will turn out to be useful in other situations as well.

We now give an outline of the paper. In Section 2, we present some preliminary results that will be applied in later sections. In Section 3, we investigate a symmetric $k$-algebra $A$ of dimension 9 with symmetrising linear form $\lambda$, over an algebraically closed field $k$ of characteristic 3 . We suppose that $A$ is local with radical $J:=J(A)$ and socle $S:=S(A)$. We assume that $Z:=Z(A)$ is isomorphic to $Z(C)$ where $C$ is as above, and that $a^{3} \in K:=K(A)$, for $a \in J$. We will see that there are several possibilities for $A$, given by Proposition 3.2, Proposition 3.4 (and Remark 3.5) and Proposition 3.6. The algebras in Proposition 3.2 and Proposition 3.6 are both unique (up to isomorphism) while the algebra in Proposition 3.4 depends on a parameter $\gamma \in k$.

In Section 4 we will see that the unique simple module for the algebra in Proposition 3.2 has infinite complexity, and in Section 5 we will prove the same thing for the algebra in Proposition 3.6. So these two algebras cannot come from blocks of finite groups. Thus our block $B$ has to be Morita equivalent to one of the algebras in Proposition 3.4 (and Remark 3.5). We also show that the unique simple module for the algebras in Proposition 3.4 has complexity 2. Hence these algebras cannot 
be distinguished by complexity arguments. Thus at this point one has to fall back on the arguments in [3], making use of the algebraic group $\operatorname{Aut}(A)$.

\section{Preliminaries}

Let $k$ be an algebraically closed field and let $R$ be a finite-dimensional $k$-algebra. We denote by $Z(R)$ the center of $R$ and by $K(R)=[R, R]$ the subspace of $R$ generated by all elements $[r, s]=r s-s r(r, s \in R)$. Moreover, we denote by $J(R)$ the (Jacobson) radical and by $S(R)$ the (left) socle of $R$. If $R$ is symmetric then $S(R)$ is also the right socle of $R$. An ideal $I$ will always be a two-sided ideal. In this case we write $I \unlhd R$. In this section we collect some known results that will be used in the sequel.

Lemma 2.1. Let $I$ be an ideal in $R$, and let $m, n \in \mathbb{N}$ such that $m \leq n$. Suppose that $I^{n} / I^{n+1}$ is spanned by the residue classes of the products $x_{i 1} \ldots x_{i n}$ ( $i=$ $1, \ldots, d)$ where $x_{i j} \in I$ for all $i, j$. Then $I^{n+m} / I^{n+m+1}$ is spanned by the residue classes of the products $x_{j 1} \ldots x_{j m} x_{i 1} \ldots x_{i n}(i, j=1, \ldots, d)$. In particular, we have

$$
\operatorname{dim}\left(I^{n+m} / I^{n+m+1}\right) \leq\left(\operatorname{dim}\left(I^{n} / I^{n+1}\right)\right)^{2} .
$$

Proof. This is Lemma E of [6].

Lemma 2.2. Let $R$ be symmetric and local. If $\operatorname{dim}\left(J(R)^{n} / J(R)^{n+1}\right)=1$ for an $n \in \mathbb{N}$ then $J(R)^{n-1} \subseteq Z(R)$.

Proof. This is Lemma G of [6].

Lemma 2.3. Let $G$ be a finite group, and let $B$ be a block of the group algebra $k G$ with defect group $D$. Suppose that $\operatorname{char}(k)=p>0$, and denote the exponent of $D$ by $p^{e}$. Moreover, let $A$ be any k-algebra Morita equivalent to $B$. Then $a^{p^{e}} \in K(A)$ for $a \in J(A)$.

Proof. For $n \in \mathbb{N}_{0}$ we define $T_{n}(B):=\left\{x \in B: x^{p^{n}} \in K(B)\right\}$ (resp. for $A$ ). Then one can show (cf. item (9) of [8]) that $T(B):=\sum_{n=0}^{\infty} T_{n}(B)=J(B)+K(B)$ holds (resp. for $A$ ). Using this, Theorem $\mathrm{J}$ in [5] shows that $x^{p^{e}} \in K(B)$ for every $x \in J(B)$. Then Corollary 5.3 in $[2]$ implies that also $x^{p^{e}} \in K(A)$ for $x \in J(A)$.

Lemma 2.4. Let $\operatorname{char}(k)=3$, and let $C$ be the $k$-algebra with generators $x, y$ and defining relations $x^{3}=y^{3}=x y+y x=0$. Then the elements $1, x, y, x^{2}, x y, y^{2}, x^{2} y$, $x y^{2}, x^{2} y^{2}$ form a $k$-basis of $C$. Moreover, the elements $1, x^{2}, y^{2}, x^{2} y, x y^{2}, x^{2} y^{2}$ form a $k$-basis of $Z(C)$, and $J(Z(C))^{2}=k x^{2} y^{2}$. In addition, the elements $x^{2} y, x y^{2}, x^{2} y^{2}$ form a $k$-basis of $S(Z(C))$, and the elements $x y, x^{2} y, x y^{2}$ form a $k$-basis of $K(C)$. 
Proof. These assertions can be obtained by straightforward computations.

\section{Some symmetric local algebras of dimension 9}

In this section $k$ will denote an algebraically closed field of characteristic 3 , and $A$ will be a symmetric local $k$-algebra of dimension 9 , with symmetrising linear form $\lambda$. We set $J:=J(A)$ and $S:=S(A)$, and we assume that $Z:=Z(A)$ is isomorphic to $Z(C)$ where $C$ is as in Lemma 2.4. We also suppose that $a^{3} \in K:=K(A)$ for $a \in J$.

For a subspace $U$ of $A$ we set $U^{\perp}:=\{a \in A: \lambda(a U)=0\}$. It is well-known that $Z^{\perp}=K$ and $J^{\perp}=S$. Moreover, for any ideal $I$ of $A, I^{\perp}$ is the (left or right) annihilator of $I$ in $A$, and thus an ideal in $A$. We start by proving some general properties of $A$ (cf. 3.3, 3.4 and 3.5 in [3]).

Lemma 3.1. The following properties hold:

(i) $J(Z) \unlhd A, J(Z) K=0$ and $J(Z)^{2}=S$.

(ii) $J(Z)+K=J(Z)+J^{2}=J(Z)+J(Z)^{\perp}=S(Z)^{\perp} \unlhd A$ and $\operatorname{dim} S(Z)^{\perp}=6$.

(iii) $J^{2} \nsubseteq Z, \operatorname{dim} J^{2} \in\{5,6\}, \operatorname{dim} J^{3}=3, \operatorname{dim} J^{4}=1$ and $J^{5}=0$.

(iv) $a b+b a \in Z$ for $a, b \in J$; in particular $a^{2} \in Z$ for $a \in J$.

(v) $J^{3}=J(Z) J=S(Z)=(K \cap Z) \oplus S \unlhd A, K \oplus S \unlhd A$ and $\operatorname{dim}(K \oplus S)=4$.

(vi) There is $a \in J$ such that $a^{3} \notin J^{4}$; in particular, $a^{2} \notin J^{3}$ and $a \notin J^{2}$.

Proof. (i) For $z \in J(Z)$, we have $Z z=k z+J(Z) z \subseteq k z+J(Z)^{2}$. Hence $\operatorname{dim} Z z \leq$ $1+\operatorname{dim} J(Z)^{2}=2$, by Lemma 2.4. Thus $\bar{A}:=A /(A z)^{\perp}$ is a symmetric local $k$-algebra, and $\operatorname{dim} \bar{A}=\operatorname{dim} A z \leq 5$. Hence Theorem B in [6] shows that $\bar{A}$ is commutative, i.e. $K \subseteq(A z)^{\perp}$ and therefore $A z K=0$.

This proves that $A J(Z) K=0$, so that $A J(Z) \subseteq K^{\perp}=Z$. Hence $A J(Z) \subseteq$ $Z \cap J=J(Z)$ i.e. $J(Z)=A J(Z) \unlhd A$, and $J(Z)^{2} \unlhd A$. Since $\operatorname{dim} J(Z)^{2}=1$ this implies that $J(Z)^{2}=S$.

(ii) Since $S(Z)=Z \cap J(Z)^{\perp}=K^{\perp} \cap J(Z)^{\perp}=(K+J(Z))^{\perp}$ we have

$$
\operatorname{dim}(K+J(Z))=\operatorname{dim} A-\operatorname{dim} S(Z)=9-3=6
$$

by Lemma 2.4. Since $S(Z) \subseteq J(Z)$, we also have $S(Z)=J(Z) \cap J(Z)^{\perp}=\left(J(Z)^{\perp}+\right.$ $J(Z))^{\perp} \unlhd A$ and thus $S(Z)^{\perp} \unlhd A$.

Now $A=k 1+J$ implies that $K=[A, A]=[J, J] \subseteq J^{2}$ and $J(Z)+K \subseteq$ $J(Z)+J^{2}$. Assume that $\operatorname{dim}\left(J(Z)+J^{2}\right)>6$. Since $\operatorname{dim} J=8$ this would imply $J=J(Z)+J^{2}+k x$ for some $x \in J$. Hence $A=Z+k x+J^{2}=Z[x]+J^{2}=Z[x]$ by Proposition 5.2 in [9]. Hence $A$ would be commutative, a contradiction.

We conclude therefore that $J(Z)+K=J(Z)+J^{2}$. 
(iii) By (i) and (ii), we have $J(Z) J^{2} \subseteq J(Z)\left(J(Z)+J^{2}\right)=J(Z)(J(Z)+K)=$ $J(Z)^{2}=S$. Thus $J(Z) J^{3}=0$ and $J^{3} \subseteq J(Z)^{\perp}$; in particular, $\operatorname{dim} J^{3} \leq 4$. By (ii), we also have $\operatorname{dim}\left(Z+J^{2}\right)=7>\operatorname{dim} Z$; in particular, $J^{2} \nsubseteq Z$. Thus Lemma 2.2 implies that $\operatorname{dim} J^{3} / J^{4} \geq 2$, so that $\operatorname{dim} J^{3} \geq 3,1 \leq \operatorname{dim} J^{4} \leq 2$ and $\operatorname{dim} J^{4} / J^{5}=1$. Hence $J^{3} \subseteq Z$ by Lemma 2.2. Thus $J^{3} \subseteq J(Z) \cap J(Z)^{\perp}=S(Z)$ by (ii); in particular, $\operatorname{dim} J^{3}=3$ by Lemma 2.4. Hence $\operatorname{dim} J^{4}=1, J^{5}=0$ and $5 \leq \operatorname{dim} J^{2} \leq 6$.

(iv) First we assume that $c^{2} \notin Z$ for some $c \in J$. In case $c \in k c^{2}+Z$ we have $c-\alpha c^{2} \in Z \cap J=J(Z)$ for some $\alpha \in k$. Hence multiplication with $(1-\alpha c)^{-1}$ yields the contradiction $c \in J(Z)$. Thus we must have $c \notin k c^{2}+Z$; in particular, $\operatorname{dim}\left(k c+k c^{2}+Z\right)=8$. We write $A=k c+k c^{2}+k d+Z$ with $d \in A$ and obtain the contradiction $K=\left[k c+k c^{2}+k d, k c+k c^{2}+k d\right]=k[c, d]+k\left[c^{2}, d\right]$. Thus $c^{2} \in Z$ for $c \in J$. Hence $a b+b a=(a+b)^{2}-a^{2}-b^{2} \in Z$ for $a, b \in J$.

(v) By (i), we have $J(Z)^{2} J=S J=0$, so that $J(Z) J \subseteq J(Z)^{\perp} \cap J(Z)=S(Z)$ by (ii). In the proof of (iii), we observed that $J^{3} \subseteq S(Z)$. Since $\operatorname{dim} J^{3}=3=\operatorname{dim} S(Z)$ this implies $J^{3}=S(Z)$. We write $J=k x+k y+J(Z)+J^{2}$ with $x, y \in J$. Then

$$
K=\left[k x+k y+J^{2}, k x+k y+J^{2}\right] \subseteq k[x, y]+J^{3} \subseteq k[x, y]+J(Z)=k x y+J(Z)
$$

by (iv). Thus $J=k x+k y+J(Z)+K=k x+k y+k x y+J(Z)$ and

$$
J^{3} \subseteq k x^{3}+k x^{2} y+k x y x+k y x^{2}+k x y^{2}+k y x y+k y^{2} x+k y^{3}+J(Z) J .
$$

Since $x^{2}, y^{2} \in J(Z)$ we have $x^{3}, x^{2} y, y x^{2}, x y^{2}, y^{2} x, y^{3} \in J(Z) J$. Moreover, we have $x y x=(x y+y x) x-y x^{2} \in J(Z) J$, and similarly $y x y \in J(Z) J$. Hence we obtain $S(Z)=J^{3}=J(Z) J$. By (ii), we have

$$
\begin{aligned}
S(Z) & =(J(Z)+K)^{\perp}=J(Z)^{\perp} \cap K^{\perp}=(J \cap Z)^{\perp} \cap Z=\left(J^{\perp}+Z^{\perp}\right) \cap Z \\
& =(S+K) \cap Z=S+(K \cap Z)=S \oplus(K \cap Z) ;
\end{aligned}
$$

for otherwise $\operatorname{dim} S=1$ would give the contradiction $S \subseteq K \cap Z \subseteq K \subseteq \operatorname{Ker}(\lambda)$.

Since $K \cap S=0$ and $(K+S)^{\perp}=K^{\perp} \cap S^{\perp}=Z \cap J=J(Z) \unlhd A$ the assertion follows.

(vi) We assume that $a^{3} \in J^{4}=S$ for all $a \in J$. Then $a^{3} \in K \cap S=0$, i.e. $a^{3}=0$ for all $a \in J$. Hence for $a, b \in J$, (iv) implies

$0=(a \pm b)^{3}=a^{3} \pm a^{2} b \pm a b a \pm b a^{2}+a b^{2}+b a b+b^{2} a \pm b^{3}= \pm a b a \mp a^{2} b+b a b-a b^{2}$ so that $b a b=a b^{2}=b^{2} a$. Thus, in the situation above, we have $[x, x y]=x^{2} y-x y x=$ 0 and $[y, x y]=y x y-x y^{2}=0$. Hence $x y \in Z \cap J=J(Z)$, a contradiction.

Now we distinguish the two cases $\operatorname{dim} J^{2}=5$ and $\operatorname{dim} J^{2}=6$. 
Proposition 3.2. In case $\operatorname{dim} J^{2}=5$ the algebra $A$ has a $k$-basis of the form $1, x, y, z, x^{2}, x y, x^{3}, x^{2} y, x^{4}$ where $y x=-x y, y^{2}=x^{2}, z^{2}=x^{4}$ and $0=x z=z x=$ $y z=z y=x^{3} y$.

Proof. By Lemma 3.1 (vi), there is $x \in J \backslash J^{2}$ such that $x^{2} \notin J^{3}$ and $x^{3} \notin J^{4}$. Then $x \notin J(Z)+J^{2}$ since $(c+d)^{3}=c^{3}+d^{3}=0+0=0$ for $c \in J(Z), d \in J^{2}$. Let $y \in J$ such that $J=k x+k y+J(Z)+J^{2}$. Then, as in the proof of Lemma $3.1(\mathrm{v})$, we have $J=k x+k y+k x y+J(Z), A=k x+k y+k x y+Z$ and $K=k[x, y] \oplus k[x, x y] \oplus k[y, x y]$. Let $z \in J(Z)$ such that $J(Z)+J^{2}=k z+J^{2}$. Since $\operatorname{dim}\left(J^{2} \cap Z\right)=4$, we also have $J^{2} \cap Z=k x^{2} \oplus J^{3}$. Thus $J^{2}=k x^{2}+k x y+k y x+k y^{2}+J^{3}=k x y+\left(J^{2} \cap Z\right)=$ $k x^{2}+k x y+J^{3}$. Since $x y+y x \in J^{2} \cap Z$ there is $\alpha \in k$ such that $x y+y x \equiv \alpha x^{2}$ $\left(\bmod J^{3}\right)$. Setting $y^{\prime}:=y+\alpha x$ we obtain: $x y^{\prime}+y^{\prime} x=x y+y x-\alpha x^{2} \in J^{3}$. We may therefore replace $y$ by $y^{\prime}$ and thus assume that $x y+y x \in J^{3}$. (Note that this replacement does not affect the other conditions placed upon $x, y, z$.)

Since $J^{3}=k x^{3} \oplus k x^{2} y \oplus J^{4}$ by Lemma 2.1, there are $\alpha, \beta \in k$ such that $x y+$ $y x \equiv \alpha x^{3}+\beta x^{2} y\left(\bmod J^{4}\right)$. Then $x^{\prime}:=x+\beta x^{2}$ and $y^{\prime}:=y+\alpha x^{2}$ satisfy $x^{\prime} y^{\prime}+y^{\prime} x^{\prime} \equiv x y+\alpha x^{3}+\beta x^{2} y+y x+\alpha x^{3}+\beta y x^{2} \equiv 0\left(\bmod J^{4}\right)$. Hence we can replace $(x, y)$ by $\left(x^{\prime}, y^{\prime}\right)$ and thus assume that $x y+y x \in J^{4}$. (We point out again that this replacement does not affect the other conditions placed upon $x, y, z$.)

We now have $[x, y]=x y-y x \equiv 2 x y\left(\bmod J^{4}\right)$ and therefore $0=2 x^{2}[x, y]=x^{3} y$ by Lemma 3.1 (i). Hence $J^{4}=k x^{4}+k x^{3} y=k x^{4}$ by Lemma 2.1. We conclude that $x y+y x=\alpha x^{4}$ for some $\alpha \in k$. Setting $y^{\prime}:=y+\alpha x^{3}$ we obtain: $x y^{\prime}+y^{\prime} x=$ $x y+y x+\alpha x^{4}+\alpha x^{4}=0=x^{3} y^{\prime}$. We can therefore replace $y$ by $y^{\prime}$ and assume that $x y+y x=0=x^{3} y$. (We stress again that this change does not affect our other conditions on $x, y, z$. In the future, we will not mention these things explicitly.)

As before, we have $J^{3}=k x^{3} \oplus k x^{2} y \oplus k x^{4}$. Since $[y, x y]=y x y-x y^{2}=x y^{2}$ and $[y, x y] \notin S$ we conclude that $y^{2} \notin J^{3}$. On the other hand, we have $y^{2} \in$ $Z \cap J^{2}=k x^{2}+J^{3}$. Replacing $y$ by a scalar multiple, if necessary, we may assume that $y^{2} \equiv x^{2}\left(\bmod J^{3}\right)$; in particular, we have $x^{2} y^{2}=x^{4}$. Moreover, there are $\alpha, \beta, \gamma \in k$ such that $y^{2}=x^{2}+\alpha x^{3}+\beta x^{2} y+\gamma x^{4}$. Then $x y^{2}=x^{3}+\alpha x^{4}$ where $x y^{2}, x^{3} \in K$. Thus $\alpha x^{4} \in K \cap S=0$, i.e. $\alpha=0$.

Similarly, we have $y^{3}=x^{2} y+\beta x^{2} y^{2}=x^{2} y+\beta x^{4}$ where $y^{3}, x^{2} y \in K$ since $[x, x y]=x^{2} y-x y x=2 x^{2} y$. Thus $\beta x^{4} \in K \cap S=0$, i.e. $\beta=0$.

Setting $y^{\prime}:=y+\sqrt{\gamma} x y$ we obtain: $\left(y^{\prime}\right)^{2}=y^{2}+\sqrt{\gamma}\left(y x y+x y^{2}\right)+\gamma x y x y=$ $x^{2}+\gamma x^{4}-\gamma x^{2} y^{2}=x^{2}, x y^{\prime}+y^{\prime} x=x y+y x+\sqrt{\gamma}\left(x^{2} y+x y x\right)=0$ and $x^{3} y^{\prime}=0$. Hence we may replace $y$ by $y^{\prime}$ and therefore assume that $x y+y x=0=x^{3} y=y^{2}-x^{2}$. 
Now $I:=k x \oplus k y \oplus k x^{2} \oplus k x y \oplus k x^{3} \oplus k x^{2} y \oplus k x^{4} \unlhd A$. Thus $I^{\perp} \unlhd A$ and $\operatorname{dim} I^{\perp}=2$. If $a \in I \cap I^{\perp}$ then there are $\alpha, \beta, \gamma, \delta, \epsilon, \zeta, \eta \in k$ such that $a=$ $\alpha x+\beta y+\gamma x^{2}+\delta x y+\epsilon x^{3}+\zeta x^{2} y+\eta x^{4}$. Hence $0=x a=\alpha x^{2}+\beta x y+\gamma x^{3}+\delta x^{2} y+\epsilon x^{4}$, i.e. $\alpha=\beta=\gamma=\delta=\epsilon=0$. Furthermore, we have $0=a y=\zeta x^{2} y^{2}=\zeta x^{4}$, i.e. $\zeta=0$.

This shows that $I \cap I^{\perp}=S$. Thus $\operatorname{dim}\left(I+I^{\perp}\right)=7+2-1=8$, i.e. $I+I^{\perp}=J$. Let $z^{\prime} \in I^{\perp}$ such that $J=k z^{\prime}+I$. Since $z^{\prime} I=0=I z^{\prime}$ we have $z^{\prime} \in Z$. Since $J^{2} \subseteq I$ this implies that $z^{\prime} \notin J^{2}$. We may therefore replace $z$ by $z^{\prime}$ and assume that $z \in I^{\perp}$.

Then $z^{2} \in S=k x^{4}$ by Lemma 3.1 (i). In case $z^{2}=0$ we would have the contradiction $z \in J^{\perp}=S \subseteq I$. Thus we must have $z^{2} \neq 0$. Hence we replace $z$ by a scalar multiple and therefore assume that $z^{2}=x^{4}$. So we finally have a basis of the desired form.

Next we turn to the case $\operatorname{dim} J^{2}=6$.

Lemma 3.3. In case $\operatorname{dim} J^{2}=6$ there are $x, y \in J$ such that $J=k x \oplus k y \oplus J^{2}$ and $\left(x y+y x \in J^{3}\right.$ or $\left.y^{2} \in J^{3}\right)$.

Proof. By Lemma 3.1, there are $x, y \in J$ such that $x^{2} \notin J^{3}$ and $J=k x \oplus k y \oplus J^{2}$. Then

$$
J^{2}=k x^{2}+k x y+k y x+k y^{2}+J^{3}=k x y+k x^{2}+k y^{2}+k(x+y)^{2}+J^{3} .
$$

Since $\operatorname{dim} J^{2}=6$ this implies that $\operatorname{dim}\left(k x^{2}+k y^{2}+k(x+y)^{2}+J^{3}\right) \geq 5=\operatorname{dim} J(Z)$. Thus $J(Z)=k x^{2}+k y^{2}+k(x+y)^{2}+J^{3}$, by (iv) and (v) of Lemma 3.1. Replacing $y$ by $x+y$, if necessary, we may assume: $J(Z)=k x^{2}+k y^{2}+J^{3}$.

Then there are $\alpha, \beta \in k$ such that $x y+y x \equiv \alpha x^{2}+\beta y^{2}\left(\bmod J^{3}\right)$. We may assume that $\alpha \neq 0$ or $\beta \neq 0$; for otherwise the result follows. Without loss of generality, we may assume that $\alpha \neq 0$. Replacing $y$ by $\alpha^{-1} y$, if necessary, we may assume that $\alpha=1$.

In case $\beta \neq 1$ we set $x^{\prime}:=x+\sigma y$ and $y^{\prime}:=x+\tau y$ where $\sigma:=2+\sqrt{1-\beta}$ and $\tau:=2-\sqrt{1-\beta}$. Then we also have $J=k x^{\prime} \oplus k y^{\prime} \oplus J^{2}$ and

$x^{\prime} y^{\prime}+y^{\prime} x^{\prime} \equiv 2 x^{2}+(\sigma+\tau)(x y+y x)+2 \sigma \tau y^{2} \equiv 2 x^{2}+x y+y x+2 \beta y^{2} \equiv 0 \quad\left(\bmod J^{3}\right)$.

Thus we may replace $(x, y)$ by $\left(x^{\prime}, y^{\prime}\right)$ and obtain the assertion.

Finally, let $\beta=1$ and set $y^{\prime}:=y-x$. Then $J=k x \oplus k y^{\prime} \oplus J^{2}$ and $\left(y^{\prime}\right)^{2}=$ $y^{2}-y x-x y+x^{2} \in J^{3}$. Thus we can replace $y$ by $y^{\prime}$ and obtain the assertion. 
Note that $U:=\left\{a^{2}+J^{3}: a \in J\right\}$ is a linear subspace of $J^{2} / J^{3}$ in case $x y+y x \in$ $J^{3}$, but not in case $y^{2} \in J^{3}$. Thus the two cases distinguished in Lemma 3.3 are essentially different. Now we treat these two cases separately.

Proposition 3.4. Let $x, y \in J$ such that $J=k x \oplus k y \oplus J^{2}$ and $x y+y x \in J^{3}$. Choosing $x$ and $y$ appropriately, the elements $1, x, y, x^{2}, x y, y^{2}, x^{2} y, x y^{2}, x^{2} y^{2}$ form a $k$-basis of $A$ with $0=x y+y x=x^{3} y=x y^{3}=x^{3}-\beta x y^{2}=y^{3}-\gamma x^{2} y$ where $\beta \in\{0,1\}$ and $\gamma \in k$.

Proof. Our hypothesis implies that $J^{2}=k x^{2}+k x y+k y x+k y^{2}+J^{3}=k x y+J(Z)$ and $A=Z \oplus k x \oplus k y \oplus k x y$. Thus $K=k[x, y] \oplus k[x, x y] \oplus k[y, x y]$ and $K \cap Z=$ $k[x, x y] \oplus k[y, x y]$. Hence $J^{3}=(K \cap Z) \oplus S=k[x, x y] \oplus k[y, x y] \oplus S$ where $[x, x y] \equiv$ $-x^{2} y\left(\bmod J^{4}\right)$ and $[y, x y] \equiv x y^{2}\left(\bmod J^{4}\right)$. Therefore $J^{3}=k x^{2} y \oplus k x y^{2} \oplus J^{4}$. In particular, there are $\alpha, \beta \in k$ such that $x y+y x \equiv \alpha x^{2} y+\beta x y^{2}\left(\bmod J^{4}\right)$. Setting $x^{\prime}:=x+\alpha x^{2}$ and $y^{\prime}:=y+\beta y^{2}$ we obtain $J=k x^{\prime} \oplus k y^{\prime} \oplus J^{2}$ and

$$
x^{\prime} y^{\prime}+y^{\prime} x^{\prime} \equiv x y+y x+\alpha\left(x^{2} y+y x^{2}\right)+\beta\left(x y^{2}+y^{2} x\right) \equiv 0 \quad\left(\bmod J^{4}\right) .
$$

We can therefore replace $(x, y)$ by $\left(x^{\prime}, y^{\prime}\right)$ and then assume that $x y+y x \in J^{4}$.

Now $x^{3} y=x^{2} y x=-x^{3} y$ implies that $x^{3} y=0$. Thus $J^{4}=k x^{3} y+k x^{2} y^{2}=k x^{2} y^{2}$ by Lemma 2.1. In particular, there exists $\alpha \in k$ such that $x y+y x=\alpha x^{2} y^{2}$. Setting $y^{\prime}:=y+\alpha x y^{2}$ we get: $x y^{\prime}+y^{\prime} x=x y+y x+\alpha\left(x^{2} y^{2}+x^{2} y^{2}\right)=0$. Therefore we may replace $y$ by $y^{\prime}$ and assume that $x y=-y x$. Now $x y^{3}=y x y^{2}=-x y^{3}$, i.e. $x y^{3}=0$.

Since $x^{3} \in K \cap Z=k x^{2} y \oplus k x y^{2}$ there are $\alpha, \beta \in k$ such that $x^{3}=\alpha x^{2} y+\beta x y^{2}$. Then $0=x^{3} y=\alpha x^{2} y^{2}$, i.e. $\alpha=0$ and $x^{3}=\beta x y^{2}$. Replacing $y$ by $\sqrt{\beta} y$, if necessary, we may assume that $\beta \in\{0,1\}$. Similarly, there exist $\gamma, \delta \in k$ such that $y^{3}=\gamma x^{2} y+\delta x y^{2}$. Then $0=x y^{3}=\delta x^{2} y^{2}$, i.e. $\delta=0$ and $y^{3}=\gamma x^{2} y$.

Remark 3.5. (i) In case $\beta=0$ we can replace $x$ by $\sqrt{\gamma} x$, if necessary, and thus assume that $\gamma \in\{0,1\}$.

(ii) Thus let $\beta=1$. In case $\gamma=0$ we can exchange $x$ and $y$ and are then back in the case $\beta=0$. Thus we may assume $\gamma \neq 0$.

In case $\gamma=1$ we get $x^{4}=x^{2} y^{2}=y^{4}$, i.e. $x^{2}\left(x^{2}-y^{2}\right)=0=y^{2}\left(x^{2}-y^{2}\right)$. But then $x^{2}-y^{2} \in S(Z)=J^{3}$, a contradiction.

Hence we may assume that $0 \neq \gamma \neq 1$. Note that our results here differ slightly from those in [3]. The reason is a mistake in [3]; one of the changes of bases there is misleading since it is in conflict with earlier assumptions. However, the author of [3] stresses that this mistake has no influence on the correctness of the main result.

Using the fact that $(\zeta x+\eta y)^{3}=\left(\zeta^{3} \beta+\zeta \eta^{2}\right) x y^{2}+\left(\zeta^{2} \eta+\eta^{3} \gamma\right) x^{2} y$ for $\zeta, \eta \in k$ one shows that $W:=\left\{a+J^{2}: a \in J, a^{3} \in J^{4}\right\}$ is a point in case $\beta=1,0 \neq \gamma \neq 1$, 
a line in case $\beta=0, \gamma=1$ and a union of two distinct lines in case $\beta=\gamma=0$. It remains to prove that in case $\beta=1,0 \neq \gamma \neq 1$ different choices of $\gamma$ lead to non-isomorphic algebras. Thus suppose that we have two $k$-algebras $A, \tilde{A}$ with $k$-bases $1, x, y, x^{2}, x y, y^{2}, x^{2} y, x y^{2}, x^{2} y^{2}$ and $1, \tilde{x}, \tilde{y}, \tilde{x}^{2}, \tilde{x} \tilde{y}, \tilde{y}^{2}, \tilde{x} \tilde{y}^{2}, \tilde{x} \tilde{y}^{2}, \tilde{x}^{2} \tilde{y}^{2}$ respectively. Suppose also that the relations $0=x y+y x=x^{3} y=x y^{3}=x^{3}-x y^{2}=$ $y^{3}-\gamma x^{2} y$ and $0=\tilde{x} \tilde{y}+\tilde{y} \tilde{x}=\tilde{x}^{3} \tilde{y}=\tilde{x} \tilde{y}^{3}=\tilde{x}^{3}-\tilde{x} \tilde{y}^{2}=\tilde{y}^{3}-\tilde{\gamma} \tilde{x}^{2} \tilde{y}$ hold where $\gamma, \tilde{\gamma} \in k \backslash\{0,1\}$. Finally, suppose that $f: A \rightarrow \tilde{A}$ is an isomorphism of $k$-algebras, and set $J:=J(A), \tilde{J}:=J(\tilde{A})$. Using the relations $x y+y x=0$ and $\tilde{x} \tilde{y}+\tilde{y} \tilde{x}=0$ one easily gets that either $f\left(k x+J^{2}\right)=k \tilde{x}+\tilde{J}^{2}$ and $f\left(k y+J^{2}\right)=k \tilde{y}+\tilde{J}^{2}$, or $f\left(k x+J^{2}\right)=k \tilde{y}+\tilde{J}^{2}$ and $f\left(k y+J^{2}\right)=k \tilde{x}+\tilde{J}^{2}$. Then, using the relations $x^{3}-x y^{2}=0=y^{3}-\gamma x^{2} y$ and $\tilde{x}^{3}-\tilde{x} \tilde{y}^{2}=0=\tilde{y}^{3}-\tilde{\gamma} \tilde{x}^{2} \tilde{y}$ one concludes that $\gamma=\tilde{\gamma}$ in both cases.

We now consider the remaining case.

Proposition 3.6. Let $x, y \in J$ such that $J=k x \oplus k y \oplus J^{2}$ and $y^{2} \in J^{3}$. Choosing $x$ and $y$ appropriately, the elements $1, x, y, x^{2}, x y, y x, x^{3}, x y x, x^{4}$ form a $k$-basis of A with $0=y^{2}=x^{2} y=y x^{2}=y x y-x^{3}$.

Proof. Our hypothesis implies that $J^{2}=k x^{2} \oplus k x y \oplus k y x \oplus J^{3}$. As before, we conclude that $A=Z \oplus k x \oplus k y \oplus k x y$ and $K=k[x, y] \oplus k[x, x y] \oplus k[y, x y]$ where $0 \not \equiv[y, x y] \equiv y x y\left(\bmod J^{4}\right)$, i.e. $y x y \notin S$. Since $y \cdot y x y=0$ we must have $x y x y \neq 0$, so that $J^{4}=k x y x y$. Since the case $x y x \in k y x y+J^{4}$ would lead to the contradiction $x y x y=0$, we must have $J^{3}=k x y x \oplus k y x y \oplus J^{4}$.

Thus there are $\alpha, \beta \in k$ such that $x^{2} y \equiv \alpha x y x+\beta y x y\left(\bmod J^{4}\right)$. Then $0=$ $x^{2} y^{2}=\alpha x y x y$ implies that $\alpha=0$. Setting $x^{\prime}:=x-\beta y$ we obtain $\left(x^{\prime}\right)^{2} y \equiv$ $x^{2} y-\beta y x y \equiv 0\left(\bmod J^{4}\right)$. Hence we may replace $x$ by $x^{\prime}$ and assume that $x^{2} y \in J^{4}$.

Again, there are $\alpha, \beta \in k$ such that $x^{3} \equiv \alpha x y x+\beta y x y\left(\bmod J^{4}\right)$. Since $0=$ $x^{3} y=\alpha x y x y$ we conclude that $\alpha=0$. Since $J(Z)=k x^{2} \oplus k(x y+y x) \oplus J^{3}$ we must have $x^{2} \notin S(Z)$, by Lemma $3.1(\mathrm{v})$. Since $x^{2}(x y+y x)=x^{3} y+x^{2} y x=0+0=0$ this implies $x^{4} \neq 0$; in particular, we have $x^{3} \notin J^{4}$ and therefore $\beta \neq 0$. Now we can replace $y$ by $\sqrt{\beta} y$ and assume that $\beta=1$. Thus $x^{3}-y x y \in J^{4}$.

Now we have $J^{3}=k x^{3} \oplus k x y x \oplus k x^{4}$. Hence the elements $1, x, y, x^{2}, x y, y x, x^{3}, x y x$, $x^{4}$ form a basis of $A$, and there exists $\alpha \in k$ such that $x^{2} y=\alpha x^{4}$. Setting $y^{\prime}:=$ $y-\alpha x^{2}$ we have $x^{2} y^{\prime}=0$, and $x^{3}-y^{\prime} x y^{\prime}=x^{3}-y x y+\alpha x^{3} y+\alpha y x^{3}=x^{3}-y x y \in J^{4}$. We may therefore replace $y$ by $y^{\prime}$ and then assume that $\alpha=0$. Thus we now have $x^{2} y=0$.

Hence there are $\alpha, \beta \in k$ such that $y^{2} \equiv \alpha x^{3}+\beta x y x\left(\bmod J^{4}\right)$. Then $y^{3}=$ $\beta x y x y \in K \cap S=0$, so that $\beta=0$ and $y^{2} \equiv \alpha x^{3}\left(\bmod J^{4}\right)$. Suppose that $\alpha \neq 0$. 
Then we set $x^{\prime}:=\alpha x$ and $y^{\prime}:=\alpha y$. Hence we have $\left(y^{\prime}\right)^{2} \equiv \alpha^{3} x^{3} \equiv\left(x^{\prime}\right)^{3}\left(\bmod J^{4}\right)$ and $y^{\prime} x^{\prime} y^{\prime} \equiv \alpha^{3} x^{3} \equiv\left(x^{\prime}\right)^{3}\left(\bmod J^{4}\right)$ and, moreover, $\left(x^{\prime}\right)^{2} y^{\prime}=0$. Thus we can replace $(x, y)$ by $\left(x^{\prime}, y^{\prime}\right)$ and therefore assume that $\alpha=1$. Thus together we may assume that $\alpha \in\{0,1\}$.

Let $\gamma \in k$ such that $y x y=x^{3}+\gamma x^{4}$. Since $K$ contains $x^{3}$ and $[y, x y]=$ $y x y-x y^{2}=x^{3}+\gamma x^{4}-\alpha x^{4}$ we must have $(\gamma-\alpha) x^{4} \in K \cap S=0$, so that $\gamma=\alpha$.

Let $\beta \in k$ such that $y^{2}=\alpha x^{3}+\beta x^{4}$. We set $y^{\prime}:=y+\beta x y x$. Then $1, x, y^{\prime}, x^{2}$, $x y^{\prime}=x y, y^{\prime} x=y x, x^{3}, x y^{\prime} x=x y x, x^{4}$ form a basis of $A$ with $x^{2} y^{\prime}=0=x^{5}$, $\left(y^{\prime}\right)^{2}=\alpha x^{3}$ and $y^{\prime} x y^{\prime}=x^{3}+\alpha x^{4}$. Thus we may replace $y$ by $y^{\prime}$ and therefore assume that $\beta=0$.

Suppose that $\alpha=1$. We set $y^{\prime}:=y+x y+y x$. Then $1, x, y^{\prime}, x^{2}, x y^{\prime}=x y+$ $x y x, y^{\prime} x=y x+x y x, x^{3}, x y^{\prime} x=x y x, x^{4}$ form a basis of $A$ with $x^{2} y^{\prime}=0, x^{5}=0$, $\left(y^{\prime}\right)^{2}=0$ and $y^{\prime} x y^{\prime}=x^{3}$. Thus we may replace $y$ by $y^{\prime}$ and therefore assume that $\alpha=0$.

Hence we are now left with the algebras in Proposition 3.2, in Proposition 3.4 and Remark 3.5, and in Proposition 3.6. It is easy to verify that these algebras are indeed local and symmetric, satisfy $a^{3} \in K$ for $a \in J$, and have a center isomorphic to $Z(C)$. In the next sections, we are going to compute the complexity of their unique simple module.

\section{The algebra in Proposition 3.2}

The following proposition which was kindly provided by J. F. Carlson shows that the algebra $A$ of Proposition 3.2 leads to a contradiction. The argument here differs considerably from the one in [3] which made use of the outer automorphism group of the $k$-algebra $A$.

Proposition 4.1. Let $k$ be a field (of arbitrary characteristic), and let $A$ be a split local $k$-algebra such that the following conditions hold:

(1) There are $x, y, z \in J(A)$ such that $x+J(A)^{2}, y+J(A)^{2}, z+J(A)^{2}$ are $k$-linearly independent in $J(A) / J(A)^{2}$, and

(2) $x z=z x=y z=z y=0$.

Then there are a minimal projective resolution

$$
\ldots \longrightarrow P_{2} \stackrel{\varphi_{2}}{\longrightarrow} P_{1} \stackrel{\varphi_{1}}{\longrightarrow} P_{0} \stackrel{\varphi_{0}=\epsilon}{\longrightarrow} k \longrightarrow 0
$$

of the trivial $A$-module $k \cong A / J(A)$ and, for $i \in \mathbb{N}_{0}$, an $A$-basis $e_{i, 1}, \ldots, e_{i, n_{i}}$ of $P_{i}$ with the following properties: 
(a) If $i=2 m$ is even then $n_{i} \geq 2^{m}$ and $z e_{i, 1}, \ldots, z e_{i, 2^{m}} \in K_{i}:=\operatorname{Ker}\left(\varphi_{i}\right)$.

(b) If $i=2 m+1$ is odd then $n_{i} \geq 2^{m}$ and $x e_{i, 1}, y e_{i, 1}, \ldots, x e_{i, 2^{m}}, y e_{i, 2^{m}} \in K_{i}:=$ $\operatorname{Ker}\left(\varphi_{i}\right)$.

Proof. Suppose first that $i=m=0$. Then we set $P_{0}:=A$ and let $\varphi_{0}=\epsilon$ : $A \longrightarrow k$ be canonical. Moreover, we set $n_{0}:=1=2^{0}$ and $e_{0,1}:=1$. Then $\varphi_{0}: P_{0} \longrightarrow k$ is a projective cover, and $z e_{0,1} \in J(A)=K_{0}$.

Now suppose that we have already constructed $P_{0}, \ldots, P_{i}$ and $\varphi_{0}, \ldots, \varphi_{i}$ with the desired properties. If $i=2 m$ is even then $P_{i}$ has an $A$-basis $e_{i, 1}, \ldots, e_{i, n_{i}}$ such that $n_{i} \geq 2^{m}$ and $z e_{i, 1}, \ldots, z e_{i, 2^{m}} \in K_{i}$. Since $\varphi_{i}: P_{i} \longrightarrow K_{i-1}$ is a projective cover, we have $K_{i}=\operatorname{Ker}\left(\varphi_{i}\right) \subseteq J(A) P_{i}$. Thus also $J(A) K_{i} \subseteq J(A)^{2} P_{i}$. Since $z e_{i, 1}+J(A)^{2} P_{i}, \ldots, z e_{i, 2^{m}}+J(A)^{2} P_{i}$ are $k$-linearly independent in $J(A) P_{i} /$ $J(A)^{2} P_{i}$, so are $z e_{i, 1}+J(A) K_{i}, \ldots, z e_{i, 2^{m}}+J(A) K_{i}$ in $K_{i} / J(A) K_{i}$. Thus there are a projective cover $\varphi_{i+1}: P_{i+1} \longrightarrow K_{i}$ and an $A$-basis $e_{i+1,1}, \ldots, e_{i+1, n_{i+1}}$ of $P_{i+1}$ such that $n_{i+1} \geq 2^{m}$ and $\varphi_{i+1}\left(e_{i+1, j}\right)=z e_{i, j}$ for $j=1, \ldots, 2^{m}$. Hence $\varphi_{i+1}\left(x e_{i+1, j}\right)=x z e_{i, j}=0$ and $\varphi_{i+1}\left(y e_{i+1, j}\right)=y z e_{i, j}=0$ for $j=1, \ldots, 2^{m}$, and we have constructed a projective cover $\varphi_{i+1}: P_{i+1} \longrightarrow K_{i}$ with the desired properties.

Finally, if $i=2 m+1$ is odd then $P_{i}$ has an $A$-basis $e_{i, 1}, \ldots, e_{i, n_{i}}$ such that $n_{i} \geq$ $2^{m}$ and $x e_{i, 1}, y e_{i, 1}, \ldots, x e_{i, 2^{m}}, y e_{i, 2^{m}} \in K_{i}$. As before, we have $J(A) K_{i} \subseteq J(A)^{2} P_{i}$. Since $x e_{i, 1}+J(A)^{2} P_{i}, y e_{i, 1}+J(A)^{2} P_{i}, \ldots, x e_{i, 2^{m}}+J(A)^{2} P_{i}, y e_{i, 2^{m}}+J(A)^{2} P_{i}$ are $k^{-}$ linearly independent in $J(A) P_{i} / J(A)^{2} P_{i}$, so are $x e_{i, 1}+J(A) K_{i}, y e_{i, 1}+J(A) K_{i}, \ldots$, $x e_{i, 2^{m}}+J(A) K_{i}, y e_{i, 2^{m}}+J(A) K_{i}$ in $K_{i} / J(A) K_{i}$. Hence there are a projective cover $\varphi_{i+1}: P_{i+1} \longrightarrow K_{i}$ and an $A$-basis $e_{i+1,1}, \ldots, e_{i+1, n_{i+1}}$ of $P_{i+1}$ such that $n_{i+1} \geq$ $2^{m+1}$ and $\varphi_{i+1}\left(e_{i+1,2 j-1}\right)=x e_{i, j}$ and $\varphi_{i+1}\left(e_{i+1,2 j}\right)=y e_{i, j}$ for $j=1, \ldots, 2^{m}$. Therefore $\varphi_{i+1}\left(z e_{i+1,2 j-1}\right)=z x e_{i, j}=0$ and $\varphi_{i+1}\left(z e_{i+1,2 j}\right)=z y e_{i, j}=0$ for $j=$ $1, \ldots, 2^{m}$, i.e. $z e_{i+1,1}, \ldots, z e_{i+1,2^{m+1}} \in K_{i+1}=\operatorname{Ker}\left(\varphi_{i+1}\right)$. So we have constructed a projective cover $\varphi_{i+1}: P_{i+1} \longrightarrow K_{i}$ with the desired properties also in this case.

Remark 4.2. In the situation above we have $\operatorname{dim} P_{i} \geq(\operatorname{dim} A) 2^{\lfloor i / 2\rfloor}$ for $i \in \mathbb{N}_{0}$. In particular, the sequence $\left(\operatorname{dim} P_{i}\right)_{i=0}^{\infty}$ grows exponentially. Thus the algebra $A$ cannot be Morita equivalent to a block of a group algebra; for otherwise the sequence $\left(\operatorname{dim} P_{i}\right)_{i=0}^{\infty}$ would have polynomial growth since finitely generated modules over blocks of finite group algebras have finite complexity (cf. [1], for example).

In the next section, we will show that also the unique simple module over the $k$-algebra of Proposition 3.6 has infinite complexity. Thus it cannot be Morita equivalent to a block of a group algebra. 


\section{The algebra in Proposition 3.6}

In this section, $k$ will again be an algebraically closed field of characteristic 3 , and $A$ will be the $k$-algebra of Proposition 3.6. Thus $A$ has a $k$-basis $1, x, y, x^{2}, x y, y x, x^{3}$, $x y x, x^{4}$ with relations $0=y^{2}=x^{2} y=y x^{2}=y x y-x^{3}$. We are going to show that the unique simple $A$-module $A / J \cong k$ has infinite complexity. First we observe that the (left) $A$-module $A y$ is spanned over $k$ by the elements $y=1 \cdot y, x y=x \cdot y, x^{3}=$ $y x \cdot y, x^{4}=x y x \cdot y$. In particular $J^{3} \cdot y \neq 0$. We will denote a minimal projective resolution of $k$ by

$$
P_{\bullet}: \quad \ldots \longrightarrow P_{2} \stackrel{\varphi_{2}}{\longrightarrow} P_{1} \stackrel{\varphi_{1}}{\longrightarrow} P_{0} \stackrel{\varphi_{0}}{\longrightarrow} k \longrightarrow 0 .
$$

Moreover let $K_{n}:=\operatorname{Ker}\left(\varphi_{n-1}\right)$ for $n \in \mathbb{N}$ and $K_{0}:=k$. We remind the reader that for each $n \in \mathbb{N}$ the map $\varphi_{n}$ factors into a projective cover $P_{n} \longrightarrow K_{n}$ of $K_{n}$ followed by the inclusion map $K_{n} \longleftrightarrow P_{n-1}$. Hence we get the following picture with short exact sequences on the diagonals:

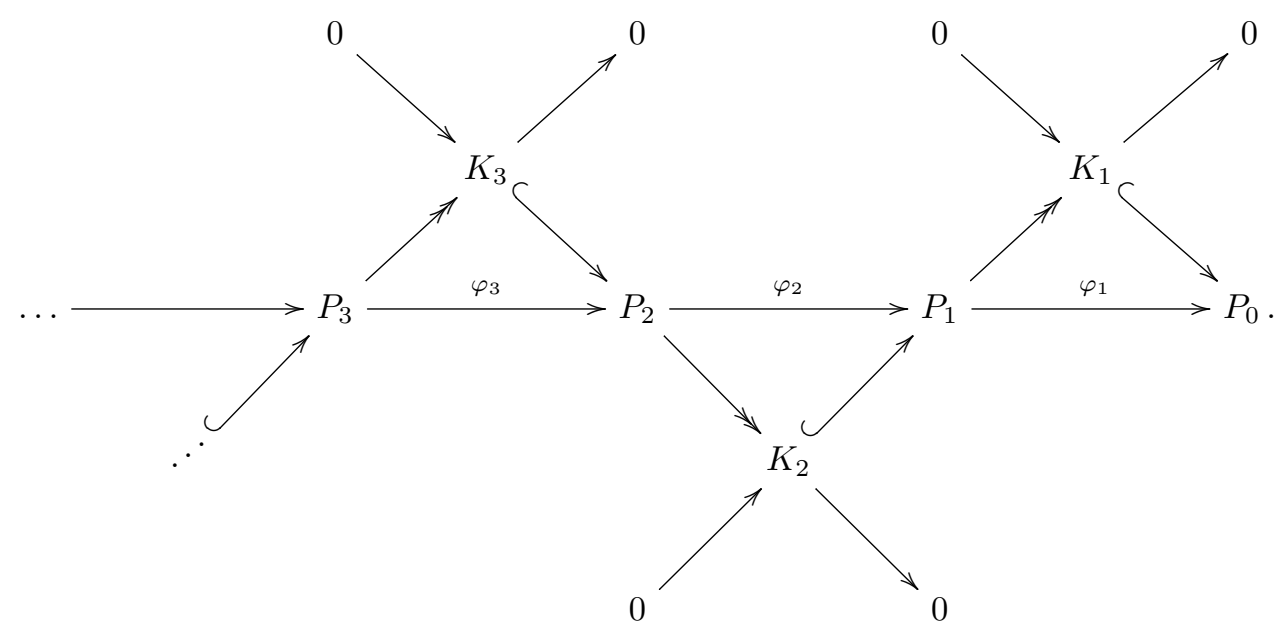

We observe that in our case, since $A$ is a local $k$-algebra, every $P_{n}$ for $n \in \mathbb{N}_{0}$ is isomorphic to a finite direct sum of copies of $A$. Moreover, since $\varphi_{0}$ can be chosen as the natural projection of $A$ onto $A / J \cong k$, we obtain $P_{0}=A, K_{1}=J$ and $J K_{1}=$ $J^{2}$. Using this we obtain $K_{1} / J K_{1}=k\left(y+J K_{1}\right) \oplus k\left(x+J K_{1}\right)$. Therefore we may choose $P_{1}:=A^{2}$. Let $B_{1}=\left\{e_{1}, e_{2}\right\}$ be an $A$-basis of $P_{1}$, i.e. $P_{1}=A e_{1} \oplus A e_{2}$. Then $\varphi_{1}: P_{1} \rightarrow P_{0}$ can be chosen to be the map defined by $\varphi_{1}\left(a_{1} e_{1}+a_{2} e_{2}\right):=a_{1} y+a_{2} x$ for $a_{1}, a_{2} \in A$. Doing so we observe that $y e_{1}, x^{2} e_{1} \in \operatorname{Ker}\left(\varphi_{1}\right)=K_{2}$ and that $\varphi_{1}\left(e_{1}\right)=y$ and $\varphi_{1}\left(e_{2}\right)=x$.

In the remaining part of this section we will inductively show that for $n \in \mathbb{N}$ the number $N_{n}$ of indecomposable direct summands of $P_{n}$ can be bounded from below 
by the $n$-th Fibonacci number (which is true for $n=1$ by the above calculations). Since the Fibonacci sequence is of exponential (and not of polynomial) growth this will show the claim we made on the complexity of the $A$-module $k$.

In view of this let us fix some $m \in \mathbb{N}$ and assume we have already constructed the following part of a minimal projective resolution of $k$ :

$$
P_{m} \stackrel{\varphi_{m}}{\longrightarrow} P_{m-1} \stackrel{\varphi_{m-1}}{\longrightarrow} \ldots \stackrel{\varphi_{2}}{\longrightarrow} P_{1} \stackrel{\varphi_{1}}{\longrightarrow} P_{0}=A \stackrel{\varphi_{0}}{\longrightarrow} k \longrightarrow 0 .
$$

As mentioned before let $N_{n}$ for $n \in \mathbb{N}$ be the number of indecomposable direct summands of $P_{n}$, i. e. $P_{n} \cong A^{N_{n}}$ for $n \in \mathbb{N}$. Let us denote by $B_{m}=\left\{e_{1}, e_{2}, \ldots, e_{N_{m}}\right\}$ an $A$-basis of $P_{m}$, so that we have $P_{m}=A e_{1} \oplus A e_{2} \oplus \cdots \oplus A e_{N_{m}}$. Since $\varphi_{m}$ is a projective cover of $K_{m}$ we obtain $K_{m+1}=\operatorname{Ker}\left(\varphi_{m}\right) \subseteq J P_{m}$, and therefore $J K_{m+1} \subseteq J^{2} P_{m}$. In particular the fact that $A$ has Loewy length 5 yields $J^{3} \cdot J K_{m+1} \subseteq J^{5} P_{m}=0$. That means every element of $J K_{m+1}$ is necessarily annihilated by $J^{3}$. Finally let $\alpha_{m}, \beta_{m} \in \mathbb{N}_{0}$ with $\beta_{m} \leq \alpha_{m}$ be such that the following conditions hold:

(i) $y e_{1}, y e_{2}, \ldots, y e_{\alpha_{m}} \in K_{m+1}$.

(ii) $x^{2} e_{1}, x^{2} e_{2}, \ldots, x^{2} e_{\beta_{m}} \in K_{m+1}$.

(iii) There exists an $A$-basis $B_{m-1}=\left\{d_{1}, \ldots, d_{N_{m-1}}\right\}$ of $P_{m-1}$ with the following two properties:

- $\varphi_{m}\left(e_{i}\right)=y d_{i}$ for $i=1, \ldots, \beta_{m}$.

- If for $i>\beta_{m}$ we have $\varphi_{m}\left(e_{i}\right)=\sum_{j=1}^{N_{m-1}} a_{i, j} d_{j}$ with $a_{i, j} \in J$ for $j=$ $1, \ldots, N_{m-1}$, then for $j=1, \ldots, \beta_{m}$ we even have $a_{i, j} \in k x+J^{2}$.

The third item from the above list might seem quite confusing at start. The essence of it is that we obtain restrictions on the occurring coefficients $a_{i, j}$ in the decomposition of image elements of $\varphi_{m}$ with regard to $B_{m-1}$ for certain preimages. Note that we may always assume that $a_{i, j} \in J$ since we have $\operatorname{Im}\left(\varphi_{m}\right)=\operatorname{Ker}\left(\varphi_{m-1}\right) \subseteq$ $J P_{m-1}=\bigoplus_{j=1}^{N_{m-1}}\left(J d_{j}\right)$.

From our calculations before one readily checks that conditions (i)-(iii) hold for $m=1$ with $\alpha_{1}=\beta_{1}=1$ (take $B_{0}=\{1\}$ and $B_{1}=\left\{e_{1}, e_{2}\right\}$ in the notation from before). Next we will check that under the assumptions (i)-(iii) we have $y e_{1}, y e_{2}, \ldots, y e_{\alpha_{m}} \notin J K_{m+1}$ and $x^{2} e_{1}, x^{2} e_{2}, \ldots, x^{2} e_{\beta_{m}} \notin J K_{m+1}$. As we remarked in the beginning of this section, we have $J^{3} \cdot y e_{i} \neq 0$ for each $i=1, \ldots, \alpha_{m}$. Since, on the other hand, we noticed that $J^{3}$ annihilates $J K_{m+1}$ we immediately get that $y e_{i} \notin J K_{m+1}$ for $i=1, \ldots, \alpha_{m}$. Hence it remains to show that $x^{2} e_{1}, x^{2} e_{2}, \ldots, x^{2} e_{\beta_{m}} \notin J K_{m+1}$. Let us therefore fix some $j \in\left\{1, \ldots, \beta_{m}\right\}$ and assume that $x^{2} e_{j} \in J K_{m+1}$. Then, in particular, we have $x^{2} e_{j} \in J K_{m+1} e_{j}$. Next we will show that this forces $x e_{j} \in K_{m+1} e_{j}+k y x e_{j}+k x y x e_{j}$. In view of this 
let us assume that $x e_{j} \notin K_{m+1} e_{j}+k y x e_{j}+k x y x e_{j}$. We have $y e_{j} \in K_{m+1} e_{j}$ by (i) and $x^{2} e_{j} \in K_{m+1} e_{j}$ by (ii). Since $A y+A x^{2}=k y+k x^{2}+k x y+k x^{3}+k x^{4}$ we obtain $\left(k y+k x^{2}+k x y+k x^{3}+k x^{4}\right) e_{j} \subseteq K_{m+1} e_{j}$. Using this and our assumption on $x$ we conclude that $x e_{j}+s \notin K_{m+1} e_{j}$ for any $s \in\left(k y+J^{2}\right) e_{j}$. For if $x e_{j}+s \in K_{m+1} e_{j}$ for some $s \in\left(k y+J^{2}\right) e_{j}$ then we could write $s=s^{\prime}+s^{\prime \prime}$ with $s^{\prime} \in\left(k y+k x^{2}+k x y+k x^{3}+k x^{4}\right) e_{j} \subseteq K_{m+1} e_{j}$ and $s^{\prime \prime} \in(k y x+k x y x) e_{j}$. Hence we had $x e_{j}+s^{\prime \prime} \in K_{m+1} e_{j}$ but then $x e_{j} \in K_{m+1} e_{j}+k y x e_{j}+k x y x e_{j}$, a contradiction. Taking into consideration the fact that $K_{m+1} \subseteq J P_{m}$ we infer that $K_{m+1} e_{j} \subseteq\left(k y+J^{2}\right) e_{j}$. But this implies that $x^{2} e_{j} \in J K_{m+1} e_{j} \subseteq\left(k x y+J^{3}\right) e_{j}$, a contradiction. This shows that $x e_{j} \in K_{m+1} e_{j}+k y x e_{j}+k x y x e_{j}$, so that there are $\xi \in K_{m+1}$ and $\lambda, \mu \in k$ with $x e_{j}=\xi e_{j}+\lambda y x e_{j}+\mu x y x e_{j}$. Hence $\xi$ is of the following form:

$$
\xi=(x-\lambda y x-\mu x y x) e_{j}+\sum_{e_{j} \neq e \in B_{m}} w_{e} e
$$

with certain $w_{e} \in J$ for $e \in B_{m} \backslash\left\{e_{j}\right\}$. Applying $\varphi_{m}$ to the element $\xi \in K_{m+1}$ and using the first part of (iii) yields

$$
0=\varphi_{m}(\xi)=\left(x y-\lambda x^{3}-\mu x^{4}\right) d_{j}+\sum_{e_{j} \neq e \in B_{m}} w_{e} \varphi_{m}(e) .
$$

In particular we obtain, by using (iii) again,

$$
\begin{aligned}
\left(x y-\lambda x^{3}-\mu x^{4}\right) d_{j} & =-\sum_{e_{j} \neq e \in B_{m}} w_{e} \varphi_{m}(e) d_{j} \\
& =-\left(\sum_{i=\beta_{m}+1}^{N_{m}} w_{e_{i}} a_{i, j}\right) d_{j}
\end{aligned}
$$

with $a_{i, j}$ being the elements we introduced in (iii) when decomposing $\varphi_{m}\left(e_{i}\right)$ into an $A$-linear combination of the elements in $B_{m-1}$. Because we have $w_{e_{i}} \in J$ and $a_{i, j} \in$ $k x+J^{2}$ for $i>\beta_{m}$ this last chain of equalities implies $x y d_{j} \in\left(k x^{2}+k y x+J^{3}\right) d_{j}$, clearly a contradiction. This shows that

- $y e_{1}, y e_{2}, \ldots, y e_{\alpha_{m}} \in K_{m+1} \backslash J K_{m+1}$, and

- $x^{2} e_{1}, x^{2} e_{2}, \ldots, x^{2} e_{\beta_{m}} \in K_{m+1} \backslash J K_{m+1}$.

If we can now show that the cosets of $y e_{1}, y e_{2}, \ldots, y e_{\alpha_{m}}$ and $x^{2} e_{1}, x^{2} e_{2}, \ldots, x^{2} e_{\beta_{m}}$ are linearly independent in $K_{m+1} / J K_{m+1}$, then the factor module $K_{m+1} / J K_{m+1}$ has a $k$-basis consisting of the union of the cosets of the elements $y e_{1}, \ldots, y e_{\alpha_{m}}$, $x^{2} e_{1}, \ldots, x^{2} e_{\beta_{m}}$ and those of some other elements $r_{1}, \ldots, r_{l} \in K_{m+1}$ with $l \in \mathbb{N}_{0}$. 
Let us therefore assume that

$$
\sum_{i=1}^{\alpha_{m}} \lambda_{i} y e_{i}+\sum_{i=1}^{\beta_{m}} \mu_{i} x^{2} e_{i} \in J K_{m+1}
$$

for some $\lambda_{1}, \ldots, \lambda_{\alpha_{m}}, \mu_{1}, \ldots, \mu_{\beta_{m}} \in k$. Multiplying this inclusion with $x y x$ and using that $J^{3}$ annihilates $J K_{m+1}$ we obtain:

$$
\sum_{i=1}^{\alpha_{m}} \lambda_{i} x^{4} e_{i}=0
$$

and hence $\lambda_{1}=\cdots=\lambda_{\alpha_{m}}=0$ since the $e_{i}$ are $A$-linearly independent. Thus we have

$$
\sum_{i=1}^{\beta_{m}} \mu_{i} x^{2} e_{i} \in J K_{m+1} .
$$

Multiplying this with $e_{j}$ from the right we obtain $\mu_{j} x^{2} e_{j} \in J K_{m+1} e_{j}$ for $j=$ $1, \ldots, \beta_{m}$. But now, apart from the coefficient $\mu_{j}$, we are in the exact same situation as before when we were indirectly showing that $x^{2} e_{j} \notin J K_{m+1}$. Using the same argument as in that case shows that $\mu_{j}$ must be zero since else we obtain a contradiction. Hence $\mu_{1}=\cdots=\mu_{\beta_{m}}=0$ and this shows that we can write

$$
K_{m+1} / J K_{m+1}=k \overline{y e_{1}} \oplus \cdots \oplus k \overline{y e_{\alpha_{m}}} \oplus k \overline{x^{2} e_{1}} \oplus \cdots \oplus k \overline{x^{2} e_{\beta_{m}}} \oplus k \overline{r_{1}} \oplus \cdots \oplus k \overline{r_{l}},
$$

where for $s \in K_{m+1}$ we denote the coset $s+J K_{m+1}$ by $\bar{s}$.

Since $B_{m}$ is an $A$-basis of $P_{m}$ and $r_{1}, \ldots, r_{l} \in K_{m+1} \subseteq J P_{m}=\bigoplus_{j=1}^{N_{m}}\left(J e_{j}\right)$ we can write

$$
r_{i}=\sum_{j=1}^{N_{m}} b_{i, j} e_{j}
$$

with $b_{i, j} \in J$ for $i=1, \ldots, l$ and $j=1, \ldots, N_{m}$. Writing $b_{i, j}=b_{i, j}^{y} y+b_{i, j}^{\prime}$ with $b_{i, j}^{y} \in k$ and $b_{i, j}^{\prime} \in k x+J^{2}$ for $i=1, \ldots, l$ and $j=1, \ldots, \alpha_{m}$ we may replace $r_{i}$ by $r_{i}-\sum_{j=1}^{\alpha_{m}} b_{i, j}^{y} y e_{j}$ for $i=1, \ldots, l$. On the level of cosets this just induces a change of basis of $K_{m+1} / J K_{m+1}$. We may hence assume that the $A$-coefficients $b_{i, j}$ belong to $k x+J^{2}$ for $i=1, \ldots, l$ and $j=1, \ldots, \alpha_{m}$.

We can now construct a projective cover $P_{m+1}$ of $K_{m+1}$. For this we set $N_{m+1}:=$ $\alpha_{m}+\beta_{m}+l$ and $P_{m+1}:=A^{N_{m+1}}$. Note that $N_{m+1} \geq \alpha_{m}+\beta_{m}$. Fixing some $A$-basis $B_{m+1}=\left\{f_{1}, f_{2}, \ldots, f_{N_{m+1}}\right\}$ of $P_{m+1}$ we have $P_{m+1}=A f_{1} \oplus \cdots \oplus A f_{\alpha_{m}} \oplus A f_{\alpha_{m}+1} \oplus \cdots \oplus A f_{\alpha_{m}+\beta_{m}} \oplus A f_{\alpha_{m}+\beta_{m}+1} \oplus \cdots \oplus A f_{N_{m+1}}$ 
We obtain a projective cover $\varphi_{m+1}: P_{m+1} \rightarrow K_{m+1}$ by setting

$$
\begin{aligned}
\varphi_{m+1}\left(f_{i}\right) & :=y e_{i} \text { for } i=1, \ldots, \alpha_{m}, \\
\varphi_{m+1}\left(f_{\alpha_{m}+i}\right) & :=x^{2} e_{i} \text { for } i=1, \ldots, \beta_{m}, \\
\varphi_{m+1}\left(f_{\alpha_{m}+\beta_{m}+i}\right) & :=r_{i} \text { for } i=1, \ldots, l,
\end{aligned}
$$

and extending this $A$-linearly to all of $P_{m+1}$. From this we immediately infer:

$$
\begin{aligned}
\varphi_{m+1}\left(y f_{i}\right) & =y^{2} e_{i}=0 \text { for } i=1, \ldots, \alpha_{m}, \\
\varphi_{m+1}\left(y f_{i}\right) & =\left(y x^{2}\right) e_{i-\alpha_{m}}=0 \text { for } i=\alpha_{m}+1, \ldots, \alpha_{m}+\beta_{m}, \\
\varphi_{m+1}\left(x^{2} f_{i}\right) & =\left(x^{2} y\right) e_{i}=0 \text { for } i=1, \ldots, \alpha_{m},
\end{aligned}
$$

so that

$$
\begin{aligned}
y f_{1}, y f_{2}, \ldots, y f_{\alpha_{m}+\beta_{m}} & \in K_{m+2} \text { and } \\
x^{2} f_{1}, x^{2} f_{2}, \ldots, x^{2} f_{\alpha_{m}} & \in K_{m+2}
\end{aligned}
$$

hold. In view of the definition of $\alpha_{m}$ and $\beta_{m}$ we set $\alpha_{m+1}:=\alpha_{m}+\beta_{m}$ and $\beta_{m+1}:=\alpha_{m}$. Then conditions (i) and (ii) are fulfilled for $m+1$ instead of $m$ and $B_{m+1}$ instead of $B_{m}$. Using the "old" $A$-basis $B_{m}=\left\{e_{1}, e_{2}, \ldots, e_{N_{m}}\right\}$ of $P_{m}$ and $\beta_{m+1}=\alpha_{m}$ we see that also (iii) holds for $m+1$ :

- By definition of $\varphi_{m+1}$ we have $\varphi_{m+1}\left(f_{i}\right)=y e_{i}$ for $i=1, \ldots, \alpha_{m}$.

- For $i=1, \ldots, \beta_{m}$ we have, by definition, $\varphi_{m+1}\left(f_{\alpha_{m}+i}\right)=x^{2} e_{i}$ and $x^{2} \in$ $k x+J^{2}$ for every such $i$. For $i=1, \ldots, l$ we have, again by definition, $\varphi_{m+1}\left(f_{\alpha_{m}+\beta_{m}+i}\right)=r_{i}$. But we had written $r_{i}=\sum_{j=1}^{N_{m}} b_{i, j} e_{j}$ and chosen the $r_{i}$ so that $b_{i, j} \in k x+J^{2}$ for $j=1, \ldots, \alpha_{m}$.

Now we are in the same situation as before and we can apply induction. Hence we have $\alpha_{1}=1, \beta_{1}=1$ and for any $n \in \mathbb{N}$ :

$$
\begin{aligned}
& \alpha_{n+1}=\alpha_{n}+\beta_{n}, \\
& \beta_{n+1}=\alpha_{n}, \\
& N_{n+1} \geq \alpha_{n}+\beta_{n} .
\end{aligned}
$$

Since the sequence $\left(\alpha_{n}\right)_{n=1}^{\infty}$ is the shifted Fibonacci sequence by the upper recursion, and we have $N_{n+1} \geq \alpha_{n}$ for $n \in \mathbb{N}$, the claim on the number of indecomposable summands of $P_{n}$ follows. We have thus shown:

Proposition 5.1. Let $A$ be the k-algebra of Proposition 3.6. Then the unique simple A-module has infinite complexity. 


\section{The algebras in Proposition 3.4}

In this section, $k$ will again be an algebraically closed field of characteristic 3, and $A$ will be one of the $k$-algebras in Proposition 3.4. Thus $A$ has a $k$-basis $1, x, y, x^{2}, x y, y^{2}, x^{2} y, x y^{2}, x^{2} y^{2}$ with relations $x y+y x=0, x^{3} y=0, x y^{3}=0, x^{3}=$ $\beta x y^{2}, y^{3}=\gamma x^{2} y$ where $\beta, \gamma \in k$. By Remark 3.5 we may assume that one of the following holds:

(I) $\beta=0$ and $\gamma \in\{0,1\}$, or

(II) $\beta=1$ and $\gamma \in k \backslash\{0,1\}$.

For these types of symmetric algebras we want to calculate the complexity of the trivial $A$-module $k$ which is also the maximal possible complexity occurring among all finite dimensional $A$-modules. We will show even more, namely:

Proposition 6.1. The unique simple $A$-module $A / J \cong k$ has complexity 2 given that $\beta \gamma \neq 1$.

Since in either of the cases (I) and (II) we always have $\beta \gamma \neq 1$ this will show:

Corollary 6.2. The unique simple $A$-module $A / J \cong k$ has complexity 2 in both case (I) and case (II).

Before we start we observe the following:

Remark 6.3. (i) The case $\beta=\gamma=0$ actually leads to an algebra which is Moritaequivalent to a block of a group algebra with defect group $C_{3}^{2}$. It is well known that for this block the maximal complexity is 2 .

(ii) Assuming $\beta \gamma \neq 1$ we can leave out the two relations $x^{3} y=0$ and $x y^{3}=0$ in the definition of $A$ at the beginning of this section since these follow from the relations $x^{3}=\beta x y^{2}$ and $y^{3}=\gamma x^{2} y$. For we have $x^{3} y=\beta x y^{3}=\beta \gamma x^{3} y$ and $x y^{3}=\gamma x^{3} y=\gamma \beta x y^{3}$. Bringing everything on one side and dividing by $1-\beta \gamma$ in each of these two equations yields $x^{3} y=0$ resp. $x y^{3}=0$.

Moreover we observe that the given relations on $A$ imply the following additional identities which will frequently be used later on:

$$
\begin{aligned}
& x\left(x^{2}-\beta y^{2}\right)=\left(x^{2}-\beta y^{2}\right) x=0, \\
& y\left(x^{2}-\beta y^{2}\right)=\left(x^{2}-\beta y^{2}\right) y=(1-\beta \gamma) x^{2} y, \\
& x\left(\gamma x^{2}-y^{2}\right)=\left(\gamma x^{2}-y^{2}\right) x=(\beta \gamma-1) x y^{2}, \\
& y\left(\gamma x^{2}-y^{2}\right)=\left(\gamma x^{2}-y^{2}\right) y=0 .
\end{aligned}
$$


Now we will calculate the first two terms of a minimal projective resolution

$$
P_{\bullet}: \quad \ldots \longrightarrow P_{2} \stackrel{\varphi_{2}}{\longrightarrow} P_{1} \stackrel{\varphi_{1}}{\longrightarrow} P_{0} \stackrel{\varphi_{0}}{\longrightarrow} k \longrightarrow 0
$$

of the trivial $A$-module $k$. In order to do this we consider the short exact sequence

$$
0 \longrightarrow K_{1}=J \longrightarrow A \stackrel{\varphi_{0}}{\longrightarrow} K_{0}=k \longrightarrow 0
$$

where $\varphi_{0}$ is just the canonical projection of $A$ onto $A / J \cong k$. Clearly $K_{1} / J K_{1}=$ $k \bar{x} \oplus k \bar{y}$, where $\bar{x}$ resp. $\bar{y}$ are the images of $x$ resp. $y$ in $K_{1} / J K_{1}$. Using this we get the second term of the minimal projective resolution by considering the short exact sequence

$$
0 \longrightarrow K_{2} \longrightarrow A^{2} \stackrel{\varphi_{1}}{\longrightarrow} K_{1} \longrightarrow 0
$$

Let $e_{1}, e_{2}$ denote the standard $A$-basis of $P_{1}:=A^{2}$. Then $\varphi_{1}$ may be defined by the rule $a_{1} e_{1}+a_{2} e_{2} \mapsto a_{1} x+a_{2} y$ for $a_{1}, a_{2} \in A$. With $\operatorname{dim}\left(K_{2}\right)=\operatorname{dim}\left(A^{2}\right)-$ $\operatorname{dim}\left(K_{1}\right)=10$ and a short calculation we easily see that $K_{2}$ has the $k$-basis $\left(x^{2}-\beta y^{2}\right) e_{1}, x^{2} y e_{1}, x^{2} y^{2} e_{1},\left(\gamma x^{2}-y^{2}\right) e_{2}, x y^{2} e_{2}, x^{2} y^{2} e_{2}, y e_{1}+x e_{2}, x y e_{1}+x^{2} e_{2}, y^{2} e_{1}-$ $x y e_{2}, x y^{2} e_{1}-x^{2} y e_{2}$ and using $J=A x+A y$ we obtain $K_{2} / J K_{2}=k \overline{\left(x^{2}-\beta y^{2}\right) e_{1}} \oplus$ $k \overline{\left(\gamma x^{2}-y^{2}\right) e_{2}} \oplus k \overline{y e_{1}+x e_{2}}$.

With the notation given above we will now continue to define projective covers $\varphi_{n}$ for $n \geq 2$ and therefore compute a complete minimal projective resolution of $k$. Doing this we will obtain that for each $n \geq 0$ the projective module $P_{n}$ can be chosen to be $A^{n+1}$. Therefore there is a minimal projective resolution of $k$ of the form

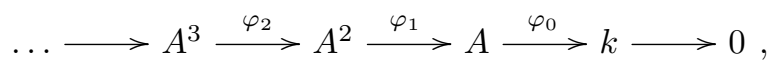

and this shows that $k$ has complexity 2 .

For now let $n \geq 1$ be a fixed natural number. Denote the standard $A$-basis of $A^{n+1}$ by $e_{1}, \ldots, e_{n+1}$ and the standard $A$-basis of $A^{n}$ by $d_{1}, \ldots, d_{n}$. We can define the map $\varphi_{n}: A^{n+1} \rightarrow A^{n}$ by defining it on the set $\left\{e_{1}, \ldots, e_{n+1}\right\}$ and extending this $A$-linearly to all of $A^{n+1}$. We set $\varphi_{n}\left(e_{i}\right):=e_{i}^{\prime}$ for $i=1, \ldots, n+1$ where $e_{1}^{\prime}, \ldots, e_{n+1}^{\prime} \in A^{n}$ are defined as follows: 
(1) If $n=4 q$ for some $q \in \mathbb{N}$ then

$$
\begin{aligned}
e_{1}^{\prime} & :=\left(x^{2}-\beta y^{2}\right) d_{1} & & \\
e_{2}^{\prime} & :=\left(\gamma x^{2}-y^{2}\right) d_{2} & & \\
e_{4 m+3}^{\prime} & :=y d_{4 m+1}-x d_{4 m+3} & & \text { for } m=0, \ldots, q-1 \\
e_{4 m+4}^{\prime} & :=x d_{4 m+2}-y d_{4 m+4} & & \text { for } m=0, \ldots, q-1 \\
e_{4 m+5}^{\prime} & :=\left(\gamma x^{2}-y^{2}\right) d_{4 m+3}+\left(x^{2}-\beta y^{2}\right) d_{4 m+5} & & \text { for } m=0, \ldots, q-2 \\
e_{4 m+6}^{\prime} & :=\left(x^{2}-\beta y^{2}\right) d_{4 m+4}+\left(\gamma x^{2}-y^{2}\right) d_{4 m+6} & & \text { for } m=0, \ldots, q-2 \\
e_{4 q+1}^{\prime} & :=\left(\gamma x^{2}-y^{2}\right) d_{4 q-1}+(-1)^{q}\left(x^{2}-\beta y^{2}\right) d_{4 q} & &
\end{aligned}
$$

(2) If $n=4 q+1$ for some $q \in \mathbb{N}$ then

$$
\begin{aligned}
e_{1}^{\prime} & :=x d_{1} & & \\
e_{2}^{\prime} & :=y d_{2} & & \\
e_{4 m+3}^{\prime} & :=y d_{4 m+1}-\left(x^{2}-\beta y^{2}\right) d_{4 m+3} & & \text { for } m=0, \ldots, q-1 \\
e_{4 m+4}^{\prime} & :=x d_{4 m+2}-\left(\gamma x^{2}-y^{2}\right) d_{4 m+4} & & \text { for } m=0, \ldots, q-1 \\
e_{4 m+5}^{\prime} & :=\left(\gamma x^{2}-y^{2}\right) d_{4 m+3}+x d_{4 m+5} & & \text { for } m=0, \ldots, q-1 \\
e_{4 m+6}^{\prime} & :=\left(x^{2}-\beta y^{2}\right) d_{4 m+4}+y d_{4 m+6} & & \text { for } m=0, \ldots, q-2 \\
e_{4 q+2}^{\prime} & :=\left(x^{2}-\beta y^{2}\right) d_{4 q}+(-1)^{q} y d_{4 q+1} & &
\end{aligned}
$$

(3) If $n=4 q+2$ for some $q \in \mathbb{N}_{0}$ then

$$
\begin{aligned}
e_{1}^{\prime} & :=\left(x^{2}-\beta y^{2}\right) d_{1} & & \\
e_{2}^{\prime} & :=\left(\gamma x^{2}-y^{2}\right) d_{2} & & \\
e_{4 m+3}^{\prime} & :=y d_{4 m+1}+x d_{4 m+3} & & \text { for } m=0, \ldots, q-1 \\
e_{4 m+4}^{\prime} & :=x d_{4 m+2}+y d_{4 m+4} & & \text { for } m=0, \ldots, q-1 \\
e_{4 m+5}^{\prime} & :=\left(\gamma x^{2}-y^{2}\right) d_{4 m+3}+\left(x^{2}-\beta y^{2}\right) d_{4 m+5} & & \text { for } m=0, \ldots, q-1 \\
e_{4 m+6}^{\prime} & :=\left(x^{2}-\beta y^{2}\right) d_{4 m+4}+\left(\gamma x^{2}-y^{2}\right) d_{4 m+6} & & \text { for } m=0, \ldots, q-1 \\
e_{4 q+3}^{\prime} & :=y d_{4 q+1}+(-1)^{q} x d_{4 q+2} & &
\end{aligned}
$$


(4) If $n=4 q+3$ for some $q \in \mathbb{N}_{0}$ then

$$
\begin{aligned}
e_{1}^{\prime} & :=-x d_{1} & & \\
e_{2}^{\prime} & :=-y d_{2} & & \\
e_{4 m+3}^{\prime} & :=y d_{4 m+1}-\left(x^{2}-\beta y^{2}\right) d_{4 m+3} & & \text { for } m=0, \ldots, q \\
e_{4 m+4}^{\prime} & :=x d_{4 m+2}-\left(\gamma x^{2}-y^{2}\right) d_{4 m+4} & & \text { for } m=0, \ldots, q-1 \\
e_{4 m+5}^{\prime} & :=\left(\gamma x^{2}-y^{2}\right) d_{4 m+3}-x d_{4 m+5} & & \text { for } m=0, \ldots, q-1 \\
e_{4 m+6}^{\prime} & :=\left(x^{2}-\beta y^{2}\right) d_{4 m+4}-y d_{4 m+6} & & \text { for } m=0, \ldots, q-1 \\
e_{4 q+4}^{\prime} & :=x d_{4 q+2}-(-1)^{q}\left(\gamma x^{2}-y^{2}\right) d_{4 q+3} & &
\end{aligned}
$$

We now show by induction that

$$
A^{n+1} \stackrel{\varphi_{n}}{\longrightarrow} A^{n} \stackrel{\varphi_{n-1}}{\longrightarrow} \ldots \stackrel{\varphi_{2}}{\longrightarrow} A^{2} \stackrel{\varphi_{1}}{\longrightarrow} A \stackrel{\varphi_{0}}{\longrightarrow} k \longrightarrow 0
$$

is part of a minimal projective resolution of $k$. For $n=1$ we have already done this above and since we have $K_{2} / J K_{2}=k \overline{\left(x^{2}-\beta y^{2}\right) e_{1}} \oplus k \overline{\left(\gamma x^{2}-y^{2}\right) e_{2}} \oplus k \overline{y e_{1}+x e_{2}}$, the claim also holds true for $n=2$. Thus let $n \geq 2$, and suppose that we have already shown that $(*)$ is part of a minimal projective resolution of $k$. We need to show that

$$
A^{n+2} \stackrel{\varphi_{n+1}}{\longrightarrow} A^{n+1} \stackrel{\varphi_{n}}{\longrightarrow} A^{n} \stackrel{\varphi_{n-1}}{\longrightarrow} \ldots \stackrel{\varphi_{2}}{\longrightarrow} A^{2} \stackrel{\varphi_{1}}{\longrightarrow} A \stackrel{\varphi_{0}}{\longrightarrow} k \longrightarrow 0
$$

is also part of a minimal projective resolution of $k$. Hence it suffices to prove that $\varphi_{n} \circ \varphi_{n+1}=0$ and that $\varphi_{n+1}: A^{n+2} \rightarrow K_{n+1}:=\operatorname{Ker}\left(\varphi_{n}\right)$ is a projective cover. In order to show these facts we distinguish the cases $(1), \ldots,(4)$. We will only consider the cases (1) and (2) since, up to some signs and the calculation of the last element $e_{n+1}^{\prime}$, case (3) is analogous to case (1) and case (4) is analogous to case (2). First we note that the exactness of $(*)$ implies that

$$
\operatorname{dim}\left(K_{n+1}\right)= \begin{cases}9 r+1 & : n=2 r-1 \text { for some } r \in \mathbb{N} \\ 9 r+8 & : n=2 r \text { for some } r \in \mathbb{N}_{0}\end{cases}
$$

which can be shown by induction.

Now let us consider case (1), i.e. $n=4 q$ for some $q \in \mathbb{N}$. Then $\operatorname{dim}\left(K_{n+1}\right)=$ $18 q+8$. One checks that the following $18 q+8$ elements are contained in $K_{n+1}$ and 
$k$-linearly independent:

$$
\begin{aligned}
x e_{1}, x^{2} e_{1}, x y e_{1}, x^{2} y e_{1}, x y^{2} e_{1}, x^{2} y^{2} e_{1}, & \\
y e_{2}, x y e_{2}, y^{2} e_{2}, x^{2} y e_{2}, x y^{2} e_{2}, x^{2} y^{2} e_{2}, & \\
x^{2} y^{2} e_{4 m+3} & (m=0, \ldots, q-1), \\
x^{2} y^{2} e_{4 m+4} & (m=0, \ldots, q-1), \\
x y e_{4 m+5}, x^{2} y e_{4 m+5}, x y^{2} e_{4 m+5}, x^{2} y^{2} e_{4 m+5} & (m=0, \ldots, q-1), \\
x y e_{4 m+6}, x^{2} y e_{4 m+6}, x y^{2} e_{4 m+6}, x^{2} y^{2} e_{4 m+6} & (m=0, \ldots, q-2), \\
y e_{1}-\left(x^{2}-\beta y^{2}\right) e_{3}, y^{2} e_{1}+(\beta \gamma-1) x^{2} y e_{3}, & \\
x e_{2}-\left(\gamma x^{2}-y^{2}\right) e_{4}, x^{2} e_{2}+(1-\beta \gamma) x y^{2} e_{4}, & \\
\left(\gamma x^{2}-y^{2}\right) e_{4 m+3}+x e_{4 m+5},(\beta \gamma-1) x y^{2} e_{4 m+3}+x^{2} e_{4 m+5} & (m=0, \ldots, q-1), \\
\left(x^{2}-\beta y^{2}\right) e_{4 m+4}+y e_{4 m+6},(1-\beta \gamma) x^{2} y e_{4 m+4}+y^{2} e_{4 m+6} & (m=0, \ldots, q-2), \\
y e_{4 m+5}-\left(x^{2}-\beta y^{2}\right) e_{4 m+7}, y^{2} e_{4 m+5}+(\beta \gamma-1) x^{2} y e_{4 m+7} & (m=0, \ldots, q-2), \\
x e_{4 m+6}-\left(\gamma x^{2}-y^{2}\right) e_{4 m+8}, x^{2} e_{4 m+6}+(1-\beta \gamma) x y^{2} e_{4 m+8} & (m=0, \ldots, q-2), \\
\left(x^{2}-\beta y^{2}\right) e_{4 q}+(-1)^{q} y e_{4 q+1},(1-\beta \gamma) x^{2} y e_{4 q}+(-1)^{q} y^{2} e_{4 q+1} . &
\end{aligned}
$$

Again, using $J=A x+A y$, we can extract the following $k$-basis of $J K_{n+1}$ consisting of $14 q+6$ elements from this list:

$$
\begin{aligned}
& x^{2} e_{1}, x y e_{1}, x^{2} y e_{1}, x y^{2} e_{1}, x^{2} y^{2} e_{1}, \\
& x y e_{2}, y^{2} e_{2}, x^{2} y e_{2}, x y^{2} e_{2}, x^{2} y^{2} e_{2} \text {, } \\
& x^{2} y^{2} e_{4 m+3} \quad(m=0, \ldots, q-1), \\
& x^{2} y^{2} e_{4 m+4} \quad(m=0, \ldots, q-1), \\
& x_{y e} e_{4 m+5}, x^{2} y e_{4 m+5}, x y^{2} e_{4 m+5}, x^{2} y^{2} e_{4 m+5} \quad(m=0, \ldots, q-1), \\
& \text { xye }_{4 m+6}, x^{2} y e_{4 m+6}, x y^{2} e_{4 m+6}, x^{2} y^{2} e_{4 m+6} \quad(m=0, \ldots, q-2), \\
& y^{2} e_{1}+(\beta \gamma-1) x^{2} y e_{3}, \\
& x^{2} e_{2}+(1-\beta \gamma) x y^{2} e_{4}, \\
& (\beta \gamma-1) x y^{2} e_{4 m+3}+x^{2} e_{4 m+5} \quad(m=0, \ldots, q-1), \\
& (1-\beta \gamma) x^{2} y e_{4 m+4}+y^{2} e_{4 m+6} \quad(m=0, \ldots, q-2), \\
& y^{2} e_{4 m+5}+(\beta \gamma-1) x^{2} y e_{4 m+7} \quad(m=0, \ldots, q-2), \\
& x^{2} e_{4 m+6}+(1-\beta \gamma) x y^{2} e_{4 m+8} \quad(m=0, \ldots, q-2), \\
& (1-\beta \gamma) x^{2} y e_{4 q}+(-1)^{q} y^{2} e_{4 q+1} \text {. }
\end{aligned}
$$


Hence the cosets of the following $4 q+2$ elements form a $k$-basis of $K_{n+1} / J K_{n+1}$ and therefore prove the proposition for case (1):

$$
\begin{array}{rlrl}
f_{1}^{\prime} & :=x e_{1}, \\
f_{2}^{\prime} & :=y e_{2}, & & \\
f_{4 m+3}^{\prime} & :=y e_{4 m+1}-\left(x^{2}-\beta y^{2}\right) e_{4 m+3} & & (m=0, \ldots, q-1), \\
f_{4 m+4}^{\prime} & :=x e_{4 m+2}-\left(\gamma x^{2}-y^{2}\right) e_{4 m+4} & (m=0, \ldots, q-1), \\
f_{4 m+5}^{\prime} & :=\left(\gamma x^{2}-y^{2}\right) e_{4 m+3}+x e_{4 m+5} & & (m=0, \ldots, q-1), \\
f_{4 m+6}^{\prime} & :=\left(x^{2}-\beta y^{2}\right) e_{4 m+4}+y e_{4 m+6} & & (m=0, \ldots, q-2), \\
f_{4 q+2}^{\prime} & :=\left(x^{2}-\beta y^{2}\right) e_{4 q}+(-1)^{q} y e_{4 q+1} . & &
\end{array}
$$

Finally we consider case (2), i.e. $n=4 q+1$ for some $q \in \mathbb{N}$. Then $\operatorname{dim}\left(K_{n+1}\right)=$ $18 q+10$ and as before one checks that the following $18 q+10$ elements are contained in $K_{n+1}$ and $k$-linearly independent:

$$
\begin{aligned}
\left(x^{2}-\beta y^{2}\right) e_{1}, x^{2} y e_{1}, x^{2} y^{2} e_{1},\left(\gamma x^{2}-y^{2}\right) e_{2}, x y^{2} e_{2}, x^{2} y^{2} e_{2}, & \\
x y^{2} e_{4 m+3}, x^{2} y^{2} e_{4 m+3}, x^{2} y e_{4 m+4}, x^{2} y^{2} e_{4 m+4} & (m=0, \ldots, q-1), \\
x^{2} y e_{4 m+5}, x^{2} y^{2} e_{4 m+5}, x y^{2} e_{4 m+6}, x^{2} y^{2} e_{4 m+6} & (m=0, \ldots, q-1), \\
y e_{1}+x e_{3}, x y e_{1}+x^{2} e_{3}, y^{2} e_{1}-x y e_{3}, x y^{2} e_{1}-x^{2} y e_{3}, & \\
x e_{2}+y e_{4}, x^{2} e_{2}+x y e_{4}, x y e_{2}-y^{2} e_{4}, x^{2} y e_{2}-x y^{2} e_{4}, & \\
\left(\gamma x^{2}-y^{2}\right) e_{4 m+3}+\left(x^{2}-\beta y^{2}\right) e_{4 m+5} & (m=0, \ldots, q-1), \\
\left(x^{2}-\beta y^{2}\right) e_{4 m+4}+\left(\gamma x^{2}-y^{2}\right) e_{4 m+6} & (m=0, \ldots, q-1), \\
y e_{4 m+5}+x e_{4 m+7}, x y e_{4 m+5}+x^{2} e_{4 m+7} & (m=0, \ldots, q-2), \\
y^{2} e_{4 m+5}-x y e_{4 m+7}, x y^{2} e_{4 m+5}-x^{2} y e_{4 m+7} & (m=0, \ldots, q-2), \\
x e_{4 m+6}+y e_{4 m+8}, x^{2} e_{4 m+6}+x y e_{4 m+8} & (m=0, \ldots, q-2), \\
x y e_{4 m+6}-y^{2} e_{4 m+8}, x^{2} y e_{4 m+6}-x y^{2} e_{4 m+8} & (m=0, \ldots, q-2), \\
y e_{4 q+1}+(-1)^{q} x e_{4 q+2}, x y e_{4 q+1}+(-1)^{q} x^{2} e_{4 q+2}, & \\
y^{2} e_{4 q+1}-(-1)^{q} x y e_{4 q+2}, x y^{2} e_{4 q+1}-(-1)^{q} x^{2} y e_{4 q+2} . &
\end{aligned}
$$


Now the following $14 q+7$ elements form a $k$-basis of $J K_{n+1}$ :

$$
\begin{aligned}
x^{2} y e_{1}, x^{2} y^{2} e_{1}, x y^{2} e_{2}, x^{2} y^{2} e_{2}, & \\
x y^{2} e_{4 m+3}, x^{2} y^{2} e_{4 m+3}, x^{2} y e_{4 m+4}, x^{2} y^{2} e_{4 m+4} & (m=0, \ldots, q-1), \\
x^{2} y e_{4 m+5}, x^{2} y^{2} e_{4 m+5}, x y^{2} e_{4 m+6}, x^{2} y^{2} e_{4 m+6} & (m=0, \ldots, q-1), \\
x y e_{1}+x^{2} e_{3}, y^{2} e_{1}-x y e_{3}, x y^{2} e_{1}-x^{2} y e_{3}, & \\
x^{2} e_{2}+x y e_{4}, x y e_{2}-y^{2} e_{4}, x^{2} y e_{2}-x y^{2} e_{4}, & \\
x y e_{4 m+5}+x^{2} e_{4 m+7} & (m=0, \ldots, q-2), \\
y^{2} e_{4 m+5}-x y e_{4 m+7}, x y^{2} e_{4 m+5}-x^{2} y e_{4 m+7} & (m=0, \ldots, q-2), \\
x^{2} e_{4 m+6}+x y e_{4 m+8} & (m=0, \ldots, q-2), \\
x y e_{4 m+6}-y^{2} e_{4 m+8}, x^{2} y e_{4 m+6}-x y^{2} e_{4 m+8} & (m=0, \ldots, q-2), \\
x y e_{4 q+1}+(-1)^{q} x^{2} e_{4 q+2}, y^{2} e_{4 q+1}-(-1)^{q} x y e_{4 q+2}, & \\
x y^{2} e_{4 q+1}-(-1)^{q} x^{2} y e_{4 q+2} . &
\end{aligned}
$$

Thus the cosets of the following $4 q+3$ elements form a $k$-basis of $K_{n+1} / J K_{n+1}$ and therefore prove case (2):

$$
\begin{array}{rlrl}
f_{1}^{\prime} & :=\left(x^{2}-\beta y^{2}\right) e_{1}, & \\
f_{2}^{\prime} & :=\left(\gamma x^{2}-y^{2}\right) e_{2}, & & \\
f_{4 m+3}^{\prime} & :=y e_{4 m+1}+x e_{4 m+3} & (m=0, \ldots, q-1), \\
f_{4 m+4}^{\prime} & :=x e_{4 m+2}+y e_{4 m+4} & (m=0, \ldots, q-1), \\
f_{4 m+5}^{\prime} & :=\left(\gamma x^{2}-y^{2}\right) e_{4 m+3}+\left(x^{2}-\beta y^{2}\right) e_{4 m+5} & (m=0, \ldots, q-1), \\
f_{4 m+6}^{\prime} & :=\left(x^{2}-\beta y^{2}\right) e_{4 m+4}+\left(\gamma x^{2}-y^{2}\right) e_{4 m+6} & (m=0, \ldots, q-1), \\
f_{4 q+3}^{\prime} & :=y e_{4 q+1}+(-1)^{q} x e_{4 q+2} . & &
\end{array}
$$

This finishes the proof.

Acknowledgement. The authors are grateful to J. F. Carlson for providing Proposition 4.1, and to S. King for several computations of projective resolutions. They also thank the referees of an earlier version of this paper for a number of useful suggestions and pay special thanks to the referees of the latest version of this paper for their input which led to significant improvements of the last two sections. 


\section{References}

[1] J. L. Alperin and L. Evens, Representations, resolutions and Quillen's dimension theorem, J. Pure Appl. Algebra, 22(1) (1981), 1-9.

[2] L. Héthelyi, E. Horváth, B. Külshammer and J. Murray, Central ideals and Cartan invariants of symmetric algebras, J. Algebra, 293(1) (2005), 243-260.

[3] R. Kessar, On blocks stably equivalent to a quantum complete intersection of dimension 9 in characteristic 3 and a case of the abelian defect group conjecture, J. London Math. Soc., 85(2) (2012), 491-510.

[4] M. Kiyota, On 3-blocks with an elementary abelian defect group of order 9, J. Fac. Sci. Univ. Tokyo Sect. IA Math., 31(1) (1984), 33-58.

[5] B. Külshammer, Bemerkungen über die Gruppenalgebra als symmetrische Algebra II, J. Algebra, 75(1) (1982), 59-69.

[6] B. Külshammer, Symmetric local algebras and small blocks of finite groups, J. Algebra, 88(1) (1984), 190-195.

[7] B. Külshammer, Crossed products and blocks with normal defect groups, Comm. Algebra, 13(1) (1985), 147-168.

[8] B. Külshammer, Group-theoretical descriptions of ring-theoretical invariants of group algebras, in Representation theory of finite groups and finite-dimensional algebras (Bielefeld, 1991), Progr. Math., 95, Birkhäuser, Basel, (1991), 425442.

[9] B. Külshammer, Lectures on Block Theory, London Mathematical Society Lecture Note Series, 161, Cambridge University Press, Cambridge, 1991.

[10] L. Puig and Y. Usami, Perfect isometries for blocks with abelian defect groups and Klein four inertial quotients, J. Algebra, 160(1) (1993), 192-225.

[11] S. Reinhardt, Eine Klasse von 3-Blöcken mit Defekt 2, Master's thesis, Jena 2015.

Burkhard Külshammer (Corresponding Author), Pierre Landrock and Stephanie Reinhardt

Institute for Mathematics

University of Jena

07737 Jena, Germany

e-mails: kuelshammer@uni-jena.de (B. Külshammer)

pierre.landrock@uni-jena.de (P. Landrock)

s.reinhardt@uni-jena.de (S. Reinhardt) 